\title{
ARGUMENT FEATURES IN NOVICE L2 WRITING. A CONTRASTIVE APPROACH
}

\author{
MARINELA BURADA* \\ Transilvania University of Brașov, Romania
}

\begin{abstract}
This paper reports findings derived from a contrastive investigation of argumentative EL (English) essays composed by non-native university students with different cultural and educational backgrounds. While the texts under analysis here have not originally been intended for comparison and contrast, approaching them in this manner made a lot of sense when a general rating revealed that, overall, one group of students fared considerably better than the other in terms of negotiating the intended message and achieving their persuasive aim, despite their being at earlier stages in their EL acquisition. The investigation has been intended for diagnostic purposes and has therefore been targeted at three problem areas in which inter-group performance discrepancies were the most conspicuous: the ways in which text writers signal their intentions and engage with the reader, the level of commitment to the thesis they advocate for, and the kind of evidence they advance in order to support it.
\end{abstract}

Keywords: academic writing, English language composition, contrastive studies, argument.

\section{INTRODUCTION}

Situated at the intersection of several different disciplines, the study of argument and argumentation has been an ongoing concern with scholars and academic writing instructors operating in different cultural, research, and educational paradigms. Situated within the frame of L2 composition pedagogy, this paper considers a number of issues identified across a small corpus of argumentative essays written in English by Romanian and Japanese students enrolled in language-related and, respectively, business study programs. Contrary to this author's initial assumption that, as English language students, the Romanian text writers would be more successful in

*m.burada@unitbv.ro 
communicating and supporting their points of view, the essays composed by their Japanese peers proved to be comparatively more readable, more persuasive, and closer to meeting their genre-related expectations. The main aim of the small-scale investigation reported below has been to determine what makes these compositions more effective, despite their authors' comparatively lower L2 proficiency. To this end, the paper has been structured as follows. Section 1 provides a few highlights in the research of argumentation, emphasizing some difficulties besetting the process of identifying, analyzing, and evaluating the argument-as-product (1.1). It briefly considers the ontology of the argument (1.2) and the external factors accounting for its variable manifestations (1.3). Against this theoretical backdrop, section 2 goes on to outline the overall design of the study, starting with the writers' profiles (2.1), the texts forming the corpus (2.2), and the analytical frame adapted for the purposes of the present analysis (2.3). Section 3 is concerned with the description of the findings in three areas: the writer's intentions (3.1), the thesis of the argument (3.2) and the evidence used by the arguers in order to bolster their claims (3.3); the following subsection (3.4), ties the strands of the discussion together and suggests possible explanations for the issues identified. Finally, the conclusions are included in section 4 .

\section{THEORETICAL PRELIMINARIES}

\subsection{Previous Research on Argumentation}

Featuring prominently among the different academic genres, essays, in general, and arguments, in particular, have been the objects of much scholarly attention over the years. Explorations carried out within different research traditions, such as informal logic and argumentation theory, philosophy, rhetoric, pragma-dialectics, composition studies, or L1 and L2 writing pedagogy, havecontributed useful data on the nature, typology, form and substance of arguments, as well as on the extralinguistic variables at work when constructing them.

Argumentation as process as well as the argumentative discourse as product have been approached from a variety of vantage points: as a dialogic relation between individuals situated on different sides of an issue (cf. Kuhn and Udell 2007), as a succession of acts i.e., of concluding, of premising, of inferring (cf. Hitchcock, 2007), as a text pattern, or as aconfiguration of specific, smaller-scale units e.g., the claim, the evidence, the warrant, etc. (cf. Toulmin 2003). A hefty amount of literature has been devoted to the most appropriate ways to recognize, understandand evaluate arguments. Recognizing arguments and distinguishing them from other types of text, 
such as narratives (Groarke 2017), illustrations (Hurley 2000), or explanations (Govier 2010, Hurley 2000, Johnson and Blair 2006) are sometimes problematic, given their conceptual relatedness. Once identified, understandingarguments entails retracing the steps in the arguers'line of reasoning; it is a principle-guided process (cf. the "principle of charity", Johnson and Blair 15) which necessarily precedes evaluation. Neither understanding, nor assessing the merits of an argument is without complications, however. One reason for this may be, for example, the difficulty to tease claims and conclusions apart, to match the form of a language unit with its intended function (e.g., when a rhetorical question serves as statement), or to separate what is essential to understanding the argument from irrelevant matter in the larger discourse (Groarke 2017). Another reasonrelates to the measure of subjectivity involved in recognizing and interpreting an argument, especially when the arguer's intentions are not signalled explicitly. From a wider perspective, the situation is further compounded by the multimodal dimension that argumentation may be taking on, such as when verbal argument is integrated or even replaced withnonverbal modes of expression e.g., visuals, sounds, smells, etc. (Hitchcock 2007, Groarke 2015).

As far as evaluation is concerned, the quality criteria meant to serve as benchmark in the assessment of arguments originate mostly from the field of logic; however, these criteria have limited validity when put into practice in educational settings (Glassner 2017). From a pedagogical perspective, investigations into the construction of written or spoken argumentshave been aimed at identifying problem areas in student performance and suggesting remedial action. This is also the vantage point on which the present study is predicated.

One obstacle in the way of connecting the dots between the knowledge stemming from the different research paradigms may be the partial overlap between what people think that an argument is or should be. A studyon L1 argument writing conducted by Wingate (2012) within the framework of EAP has shown that students and academic tutors had fuzzy notions on the nature of argumentation and on theeffectiveways to learn/teach it. Another investigation carried out by Stapleton and Wu (2015) has identified the gap that sometimes exists between the arguers' knowledge of the formal schemata - or as the researchers put it, "the surface structure of the argument" - and the quality of the arguer's reasoning. In other words, as their study has shown, knowing how to write an argumentative text does not in and by itself guarantee the successof the argument. An approach is needed, then, that would keep both the structure and the substance of persuasive texts in equal focus (Stapleton and $\mathrm{Wu}$ ). 


\subsection{The Essence of Argument}

Having touched upon some of the issues that the multidisciplinary research of argumentation has held up to light, a few comments on its nature are in order here. For the purposes of this paper, the discussion will be confined to the notion of argument-as-product.

Within the formal logic paradigm, an argument is "a group of statements, one or more of which (the premises) are claimed to provide support for, or reasons to believe, one of theothers (the conclusion)" (Hurley 1). Its primary aim is to achieve rational persuasion (Johnson 2001). Particularized to written discourse, argumentis a formal label that applies to a specific syntactic or discourse unit, i.e. a sentence, a paragraph, or to a text as a whole. From a structural point of view, then, an argument is a language unit of variable length consisting of one or more items of evidence intended to uphold a claim. Texts based on this simple pattern have been called "proto-arguments" (Groarke 2017). This is, according to Johnson (2001), the first tier of the argument, or its "illative core". Johnson takes a step further in positing that "genuine arguments" are two-tiered structures which include, besides an illative tier, a dialectical tier. This presupposes that, in making a case, the arguer is also expected to meet his/her dialectical obligations, that is, to consider alternative points of view and anticipate potential objections to his/her conclusions and premises.

This is what Kuhn and Udell call "dual-function arguments" (Kuhn and Udell 98), i.e., a construct consisting of two arguments, one for the preferred option, the other against the alternative to it. By this token, "single-function arguments" contain either one, or the other. One particularity of written arguments is, as Strongman points out, that they are produced in a context where the opponent is not present (Strongman 4). This makes it necessary for the arguer to anticipate the opponent's arguments and to represent them fairly.

\subsection{The Variables of Argument}

To amend the rather static view of the argument that the foregoing discussion may have created, it is necessary to pick up on the idea relative to the variables that shape it, mentioned above (cf. 1.1), Discussing the issue of identity in academic writing, Coffin et al. argue that student writing, among others, is influenced by "educational background, ethnicity, cultural expectations and gender". This calls for a more holistic approach to argumentation, an approach which also takes the setting of writing into account (Coffin et al. 11). This broader perspective is particularly relevant to the present investigation and is therefore worth considering in some detail. 
The main question to be addressed here is this: given the formalized pattern of the argument (cf. 1.2), how can the variations in instantiating this pattern be accounted for? In other words, what are the factors responsible for variations in the construction of argument? In a social constructivist vein, the view espoused in the present study is that audience and context hold the key to a better understanding of writing, in general.

\subsubsection{The Audience}

To begin with, it is important to note that, unlike in formal logic, argumentation constructed as part of social and professional communication isprimarily intended as apersuasive act. This presupposes the presence of an audience that persuasion is targeted at. As the context of situation varies, so does the audience. Hence the arguers' need to adapt their argument so as to tune in to the intended audience's beliefs, values, way of thinking, etc., in order to achieve their communicative goal.

\subsubsection{The Context}

In the Saussurean tradition, language does not belong to any single individual, but is the product of the community, as a whole. This social dimension has also been attributed to writing, which, as Grabe and Kaplan suggested, "can only be understood from the perspective of a social context and not as a product of a single individual" (Grabe and Kaplan 94).

In a narrower sense, context may refer to the microculture i.e., the disciplinary communitywithin which argumentation is conducted. Previous research has shown that disciplinary constraints play a determining role in shaping professional discourse, including argument writing, and that, as Wingate states, "what is accepted as a well-formed and validargument in an essay depends on the discipline's value system and epistemology, and there is great variation across disciplines.” (Wingate 146) What this means is that, to all intents and purposes, what counts as good argument in one context, may work less effectively in another.

In a wider acceptation, context may refer to the cultural setting within which argument writingoccurs. Useful insights into this matter come from the field of contrastive rhetoric. For one thing, it is worth remembering Kaplan's (1966) initial claim that

Logic (in the popular rather than the logician's sense of the word) which is the basis of rhetoric, is evolved out of culture; it is not universal. Rhetoric, then, is not universal either, but varies from culture to culture and even from time to time within a given culture. It is affected by canons of taste within a given culture at a given time. (qtd in Connor 30) 
Since argumentation involves reasoning, this contentious, much-debated view deserves some consideration: put simply, even if one isinclined to refute the idea that culture determines the outcome of a rational process, one cannot offhandedly discard the notion that culture may exert at least some influence on the process itself.

For instance, contrastive research on L1 student writing in English and Japanese (Hinds, in Connor), showed that texts composed in Japanese followed the traditional four-unit essay structure. Also, their sparing use of metalanguage made it the reader's onus to extract the intended message, which accounted for their reader-responsible prose. As further explained by Ädel, "in a reader-responsible culture like Japanese, connections between various parts of a text are more commonly left implicit” (Ädel 149). Hinds (1987, 1990, qtd in Connor 42) established a direct link between readerresponsibility and the Japanese (and Oriental, in general) tendency to delay the introduction of the thesis statement. The result is what he calls a "quasiinductive" style, a pattern that is neither deductive, nor inductive. As far as the Romanian context is concerned, it is rather unfortunate that comparable data and generalizations on L1 composition have not, as yet, seen the light of academic day.

\section{DESIGN OF THE STUDY}

It is important to state from the outset that neither the writing task, nor the resulting texts were initially intended as sources of contrastive data. The interest for a contrastive approach was sparked by the realization that the script authors seemed to hold different views on what constitutes compelling evidence and on how it can be marshalled in order to achieve its persuasive aim.

\subsection{The Text Writers}

The authors of the L2 texts under consideration here belong to two mixed gender groups of first and second year undergraduate students within the age range of 18 to 20+ years. One group consisted of nine business students from Otaru University of Commerce, Japan, whose previous instruction at their home university included a debate class in English. The other group was made up of nine Romanian students of English (major or minor) at Transilvania University of Brașov, Romania. Although the Romanian writers'exposure to English was high, they had received no formal training in EL composition. 


\subsection{The Data}

The text corpus consists of eighteen samples of novice EL writing distributed in two subcorpora, depending on the writers' native language. Throughout this paper, these subcorpora will be distinguished by the text writers' L1; they will be referred to as the Japanese corpus (or set) and, respectively, the Romanian corpus (or set).

The eighteen texts are the outcome of an essay writing competition which did not seek to rate the performance of one group of students against the other, but rather to identify the top two papers in each group. Originally, twelve Japanese and nine Romanian students entered the competition; for our present purposes, the last three papers submitted by the Japanese authors were left out, in order to balance the two sets. The writing task was tersely worded and deliberately genre-unspecific: "Consider the two views on tradition expressed below. Which point of view would you agree with? Write an essay in English giving and justifying your point of view.”

Table 1 The writing cue

"There is no creation without tradition; the 'new' is an inflection on a preceding form; novelty is always a variation on the past."

(Carlos Fuentes, Myself with Others: Selected Essays)
"Tradition kills originality; you keep repeat-ing the same things in tradition! Behave like the sky; always create new and different things; be original!”

(Mehmet Murat Ildan)

The specified length of the essay was 700 to 1,000 words. The evaluation criteria that the students were presented with targeted both linguistic and rhetorical aspects of the text: merit points were awarded for accuracy in spelling and grammar, for the appropriacy and range of the vocabulary used, as well as for the clarity, strength and soundness of the arguments supporting the text writer's point of view. The texts were composed at home and the time allocation was such as to remove time pressure and allow students to ponder upon their work.

Each text was assessed by five teachers from the participants' universities and their combined scores (maximum 450 points/paper) decided the winning scripts. For convenience, the essays in the Japanese set $(7,278$ words) have been referenced as J1 through J9, and those in the Romanian set (8,058 words) as R1 through R9. Across the entire corpus, five essays include a title (J4, R1, R4, R6, and R7), and two (J2 and R3) provide References for the internet-derived information they use as evidence. The extracts used for illustration purposes in this paper have been preserved as in 
the original, "warts and all", with sentence-level errors (typos, spelling, grammar) left uncorrected.

\subsection{The Analytical Frame}

The investigation has been aimed at collecting contrastive data relating to (1) the way in which the authors signal their intentions, specifically, the metalanguage employed in order to signal the argument and organize the propositional content, self-mentions, and writer-reader interactions; (2) the position and degree of explicitness of the thesis statement, and (3) the types of evidence supporting the arguer's case.

The micro-analysis reported in the next section makes use of the following concepts: Thesis, Claim, Support, Reason, and Evidence. The stretches of text in which they are instantiated are of variable length, going from one clause to one or more paragraphs.

In the present acceptation, a thesis is an explicit or implicit expression of the arguer's stand on the matter at issue. Thethesis is explicit when the writers commit themselves openly to one side of the argument or another, and implicit, when the arguer's position is the result of inference on the part of the reader. Implicit theses have been considered claims, discourse units or other cues (e.g., the paper's title and/or subtitle, rhetorical questions) indicating the side of the argument that the writers have placed themselves on. The Claim is an arguable assertion in the text (other than the Thesis) which may or may not be directly related to thesis; although claims are not the primary focus here, they will be referred to in connection with the type of support that authors use to bolster their theses and claims. The Support is taken here as a complex macrostructural unit consisting of a Reason for an assertion and the Evidence whereby the arguers back up their Theses, Claims, or Reasons.

Apart from these units, the texts contain, to variable degrees, nonarguments i.e., expository passages containing explanations, background information/introductions, or developments of a topic sentence.

\section{FINDINGS AND DISCUSSION}

\subsection{The Writer's Intentions}

Identifying the writers' explicit or implicit intentions is, according to Johnson and Blair (2006), tantamount to being able to ascertain whether their texts are arguments or not. Besides that, it can further be argued that the manner in which the writer's aims are signalled makes it possible to 
determine how reader-aware the author has been or, to put it differently, how writer-responsible his/her prose is.

Signalling intentions involves stating one's position on the issue under debate, on one hand, and creating expectations as to how this position will be defended, on the other. While the former will be held over to subsection 3.2 below, it is the latter aspect that concerns us here.

In the clear-cut cases, the writers' intentions are communicated with the help of metastatements. They are used by four writers in the Japanese group, predominantly for advance-labelling purposes (e.g., 1). An advance-labelling metastatement is a discourse unit whereby "the writer both labels and commits him/herself to perform a discourse act” (Tadros 75). One paper (J1) includes two metastatements, the second of which combines recapitulation and advance-labelling (e.g., 2).

(1) There are mainly three reasons and examples why I agree with the idea. I would like to show these reasons and my idea in this essay. (J5)

(2) There are three reasons why I think so. At first, I will explain how we come up with a creative idea to show you that 'novelty is always a variation on the past' is the right opinion.

$[\ldots]$

I explained something new is based on the former experiences. It is a general opinion. Next, I will show you some examples to support this general opinion. The example is the history of classical music. (J1)

In most cases, however, the writer's intentions are less explicit. When this happens, there are three clues that Johnson and Blair consider useful in the attempt to recognize the author's aims as well as different features of his/her argument: (1) the metalanguage consistent with argumentation i.e., premise and conclusion indicators; (2) the context of situation or, as the authors put it, the "habitat of the communication" (Johnson and Blair 14); and (3) the logical relations holding between different statements in the text. Since metalanguage is a significant point of discrepancy between the texts in the two sub-corpora under analysis, it deserves a closer look.

The Japanese text writers make extensive use of metalanguage. Premise and conclusion indicators are well-represented and tend to occur multiple times in each paper i.e., therefore, consequently, for these/those reasons, thus, in the light of the evidence, given this information, so, because, since, (that is) the reason why. But the functional range of the metalanguage illustrated in these papers is obviously wider than that. Without getting into too much detail and drawing mainly on the categories put forward by Vande Kopple (1985, in Connor) and Hyland (2010), it will be noted that the metadiscoursal markers identified - individual items or phrasaland clausal 
units- serve the overall purposes of organizing the propositional content (via sequencers, frame markers, code glosses), signalling other logical relations (e.g., additive, adversative, cause-effect, concessive) between different chunks of text, showing the writer's attitude and degree of commitment to the propositional content, and indicating the level of his/her engagement with the reader (e.g., via hedges, boosters, attitude markers, self-mentions). The discoursal markers listed here occur once or more than once in the nine texts in the Japanese set: however, but, nevertheless, yet, also, in addition, furthermore, what's more, on the one hand/on the other hand, first/firstly, second/secondly, thirdly, for example, for instance, this can be illustrated by the fact that..., here is one of my experiences..., according to, otherwise, then, although, despite, on the contrary, in short, this means, in other words, in my opinion, in conclusion, completely, totally, unfortunately. Six out of nine authors explicitly refer to themselves by means of the first person singular pronoun; the overall number of occurrences of pronoun $I$ and related case forms my, me is 106 . Besides the first person singular $I$, four authors also alternate between generic we and you in general statements, as well as in references to shared values or knowledge:

(3) Then, we have to judge if our work is good or bad, and if our work has originality or not. In this time, how do we judge? There is an only way. We can compare our works with former works. This is an only way to define our works. At the same time, we can define ourselves by comparing with others. How do you know about yourself? You compare you with others. [...] So, we cannot ignore others if you want to evaluate your character or your works. (J3)

More interestingly, however, the five texts in which it occurs, the pronoun you is mainly used in order to explicitly acknowledge the reader, which accounts for the interactional, dialogic tenor of the discourse:

(4) In fact, tradition can help us to make up something new. When you appreciate another aspect that tradition has, you will fully understand this quote of Carlos Fuentes [...]. To do so, now you have to take off the clothes you might have put on your own perspectives for a long time and that made you believe the bias: tradition is an enemy. (J4)

(5) Fashion and dishes are good examples. As you know, fashion repeats itself. (J7)

The Romanian writers' intentions are, by comparison, less immediately apparent to the reader. Metastatements are present in only two papers, one of which (R5) includes three separate instances located not at the beginning but in the main text and concluding unit: 
(6) To make it a little bit clearer, I will give an example.

[...]

Leaving the fields that may be considered by some people scientific and for a large discussion, I will turn attention to a simple one.

[...]

In conclusion, I would like to make a little generalization of what I've written. (R5)

As far as metalanguage is concerned, the density of metadiscoursal markers is lower than in the papers from the Japanese corpus. The argument indicators employed by the Romanian students match in variety - if not in frequency - those used by their Japanese peers: because, since, so, taking it all into consideration, thus, therefore, hence, consequently, as a result, ergo.The other markers, however, are comparatively more limited in both range and number of occurrences: however, but, nevertheless, yet, also,moreover, on the one hand/on the other hand, firstly, secondly, thirdly,first of all, for example,I will give an example, on the contrary, in other words,meaning, I mean, in my opinion,in my point of view,I am positive that. The incidence of self-mentions is much lower than in the Japanese corpus: 58 instances of $I, m y$, me occurring in seven texts. Instead, the use of generic pronouns is all-pervasive, especially of we, that occasionally alternates with generic/impersonal one and you:

(7) Tradition helps us imagine the new. Our culture, our language and our traditions are the foundations on which we build our identity. We must never forget who we were and where we came from; this is the only way we can determine who we are today and who we want to become tomorrow. We need tradition in order to evolve, to create and to embrace the new things in life [...]. (R7)

(8) Were one to ponder the assets and setbacks of both renewal and lore, comparative handling is required: Art means life. Life evens people. People engender art. Art devises beauty. Exquisiteness burgeons kindness, virtue, which in turn create love. Affection is you yourself which means heavenly quintessence. (R1)

(9) Thus, as long as you are not consciously aiming for it, $I$ wouldn't say that 'tradition kills originality', as Mehmet Murat Ildan puts it. (R4)

It becomes apparent, then, that in contrast to the texts in the Japanese corpus, these essays are predominantly writer-centred and monologic in character. 


\subsection{The Thesis: Location and Level of Explicitness}

Invariably, the Japanese writers make their position known early on by introducing the thesis statement either in the opening sentence or in the first paragraph. Regardless of its level of explicitness, the thesis unambiguously situates the writer on one side of the argument or the other. It is interesting to note that three papers in the Japanese corpus conclude by reiterating the thesis either explicitly (two cases) or implicitly (one case).

As specified in subsection 2.3 above, an explicit thesis has been considered the overt expression of the text writer's stand on the matter under debate. In six out of nine cases, the author's stand is signalled by means of explicit stance markers such as I agree, I support, which occur either on their own or in combination with boosters or hedges, indicating the writer's degree of commitment to the propositional content in the writing cue. For example,

(10a) I completely agree with the statement of Carlos Fuentes. Tradition is very important for creation, because I know it through my experience. (J3)

(10b) I think both points are understandable because each of them can see different aspects by what to focus on. But If I want to enhance my creativity, I must say that I would agree with Mehmet Murat Ildan's viewpoint of tradition. (J6)

By comparison, in three of the papers from the Japanese set the thesis statement is articulated in a less explicit or assertive manner, but the writer's position is nonetheless easily identifiable. In two texts (J7, J8), the thesis occurs in the opening line and takes on the form of a generic sentence used as a keynote statement. For example,

(11) Tradition is the key to creating something new. We cannot create the new without tradition. (J7)

And only in J4 the writer's position is suggested in the title, "Tradition: an Effective Resource for novelty" and expressed, in a more implicit manner, in the introductory paragraph:

(12) [...] tradition can help us to make up something new. When you appreciate another aspect that tradition has, you will fully understand this quote of Carlos Fuentes 'There is no creation without tradition; the $<$ new $>$ is an inflection on a preceding form; novelty is always a variation on the past'. (J4) 
The situation is quite different with the texts composed by the Romanian students. Only one script includes an explicit thesis occurring as an opening statement, in a manner similar to (11a) above. At the other end of the spectrum, one paper contains a conclusion unit from which the writer's position can be gleaned with some difficulty. And there are several situations in-between. R2 introduces an implicit thesis in the third paragraph, after a two-paragraph introductory unit - a structure consistent with the traditional Introduction-Body-Conclusion essay format. Similarly, in R4 the writer's position is inferable from the title, "Tradition - An Impetus to the Creative Process", and is stated explicitly in the second paragraph:

(13) My personal beliefs on the subject matter are strongly tied to the opinion expressed by Carlos Fuentes; [...] (R4)

In $\mathrm{R} 8$ the thesis is implicit in the opening position and explicit in the concluding unit:

(14) There is no creation without tradition Creation. A diverse range of human activities involving imaginative or tehnical [sic] skills that have existed for almost as long as humankind.

[...]

In conclusion, I agree with the poet Carlos Fuentes' statement that there is no creation without tradition and the new is just an inflection on a preceding form. (R8)

Three other papers contain only implicit theses introduced at the beginning (in one case by the title and, in another, by the subtitle) and reiterated at the end of the paper. Further, in R1 the writer's viewpoint is announced in the title (italics added) and in the opening statement, and then occurs as an explicit thesis statement in the latter half of the text:

(15) The state-of-the-art - a fledgling evanescent into the incandescence of tradition

Only the Almighty can create from nothing. We create from what already exists.

[...]

I do value the other quotation as utmost significant as, though we cannot attain anything unprecedented, completely novel, there cannot be a fictitious world without this real one, nor could we highlight the good without scolding the bad. Ergo when reckoned that «There is no creation without tradition», the 'new' being an inflection on a preceding form, and novelty always «a variation on the past», we actually acknowledge that without a given start we slightly advance. (R1) 
Overall, it has so far become apparent that the thesis statements invariably occur in front position in the texts produced by the Japanese students, and in front, middle, and/or end position in the Romanian papers. Cross-sample variation has also been found in the degree of explicitness of the writer's thesis. The incidence of overt side-taking on the part of the text writers is higher in the Japanese corpus and lower in the papers from the Romanian set, where the writer's position on the subject in hand is most often inferable, rather than stated explicitly. The binary opposition explicit versus implicit is convenient in the context of this analysis; it should nevertheless be noted that, while the former has been considered in absolute terms, evidence from the texts in both sets has shown that implicitness is a matter of degrees since some viewpoints appear to be introduced in more roundabout ways than others. Such variations have been discounted here, so that all the theses not verbalized assertively by their holders have been lumped together into the broad category of "implicit theses".

\subsection{Types of Evidence}

Another aspect worth attending to is the support that writers in each group provide in order to back up their assertions (i.e., theses, claims, and/or statements). Within the present analytical framework, the support is conceptualized as a more complex macrostructural unit made up of a reason for the author's assertion and the evidence introduced as proof. A CLAIMSUPPORT sequence is illustrated in the examples below. The reason and evidence forming the support unit may be signalled explicitly, by means of metadiscoursal markers, as the italicised items in (16), (17) and (18) show, or may be implicit, as in (19).

(16) You can make new things by ckecking former works [CLAIM] because creation is combination. [REASON] For example, ... [EVIDENCE]. (J3)

(17) There is no creation without tradition [THESIS] because we are using structures, rules and concepts that date for centuries, i.e tradition. Without these, we would not know what to do, how to do and so forth. [REASON] For example, ... [EVIDENCE]. (R2)

(18) Yet Japanese people, and more specifically Japanese men, still have the idea that men should work outside home and women should work inside. [CLAIM] Why does this happen? What caused this situation? It has a lot to do, I believe, with Japanese people's traditional idea about each gender's role in society. For centuries, Japanese people... [EVIDENCE]. In the workplace, there are other cultural reasons as well. Companies always... [EVIDENCE]. (J9)

(19) I am a sincere believer in the idea that the past breathes in every aspect we tend to call 'new' or 'unique'. [CLAIM] Ø Tradition is 
ubiquitous, it cannot be separated from anything that is being created. [REASON] Ø In spite of the fact that ... [EVIDENCE]. (R4)

However, not all the support units include both these constituents: in fact, there are more cases where the support consists exclusively of the evidence, with the reason left unstated. But given the fact that some texts (especially those composed by the Romanian students) make relatively little use of metalanguage, the possibility exists that some of the implicit reason statements may have escaped detection. It should also be noted that the relative position of the claim/thesis and the evidence varies, depending on the deductive or inductive patterning of the paragraph. The former type is instantiated in examples (16) and (17) above; in the latter case, the thesis/claim follows as a corrolary to the evidence and is typically introduced by conclusion or by inference indicators:

(20) According to Maria Popova... [Evidence]. In addition, Steve Jobs... [EVIDENCE].

For these reasons, it is true that 'novelty is always a variation of the past'. [THESIS] (J1)

(21) Firstly, I would like to give an example of Japanese food... [EVIDENCE]

Therefore I think that there is no creation without tradition. [THESIS] (J5)

(22) Nowadays, due to the efforts in programming, technology specialists have developed applications and programmes that allow us to use voice on the internet and Internet on smartphones [EVIDENCE] and, consequently, we can clearly speak of 'new' as an inflection on a preceding form, [CLAIM] namely the basic long distance communication principles developed to new level. (R2)

It is noticeable, at first blush, that the density of support units is higher in the Japanese set, which includes a total of 38 instances of evidence, as opposed to 28, in the Romanian set. This is significant, considering that the latter outnumbers the Japanese corpus by 780 words.

But beyond this quantitative discrepancy, the type of evidence that writers in each group rely on when arguing their case is more interesting. Therefore, the following account's main focus is on the nature of this evidence, rather than on its evaluation in terms of cogency or soundness. Overall, the items of evidence identified across the entire corpus fall into five broad categories, i.e. expert opinion, personal experience, illustration, fact/precedent, and personal opinion. Table 2 provides a breakdown of the number of occurrences for each category, found in each of the two text sets. 
Table 2 The Evidence: categories and number of occurrences

\begin{tabular}{|c|c|c|}
\hline Categories & Japanese group & Romanian group \\
\hline EXPERT OPINION & 7 & 2 \\
\hline PERSONAL EXPERIENCE & 3 & 0 \\
\hline ILLUSTRATION & 14 & 24 \\
\hline FACT/PRECEDENT/LOGIC & 9 & 0 \\
\hline PERSONAL OPINION & 5 & $2(?)$ \\
\hline
\end{tabular}

As the data in the table indicates, there is a considerably higher variety of evidence types in the Japanese corpus: one text only (J9) employs four items of evidence that all pertain to the same broad category i.e., fact/precedent/logic. The remaining eight essays include a variable amount of evidence falling into two (J2, J4, J5, J7), three (J1, J6) or four (J3, J8) different types. By contrast, only two essays in the Romanian corpus (R5, R8) include more than one type of evidence.

Evidence by personal opinion has been the most problematic category to deal with: on one hand, not all the authors' statements were explicitly marked as personal opinion; on the other hand, even when explicit, not just any instance of personal opinion was intended as support to a claim. Out of the five instances found in the Japanese corpus, only three were introduced overtly, by means of the viewpoint marker I think; in the other two cases, the interpretation has relied on the larger context. But whether signalled or not, personal opinions offered by the Japanese writers are invariably accompanied by some explanation or illustration aimed at allowing the readers a glimpse into what the arguer's opinionis based on. This is what makes them evidence by personal opinion, as distinct from mere opinion.

The situation is less clear-cut with the essays in the Romanian corpus. Apart from the two instances that safely fit into this category, there are good reasons to believe that the number of statements of personal opinion are actually much higher, had the authors not hidden themselves behind generic, collective we, and their own opinions, within the folds of broad sweeping statements.

Illustrations are the kind of evidence that writers from both groups prefer; Romanian students draw copiously on them but, unlike their Japanese peers, are in some cases less inclined to expand on them or indicate to the reader how the illustrations they use are relevant to the claim. A good reason for this may be the fact that, as shown earlier (cf. 3.1), these writers show relatively little reader awareness. The two extracts below are instances of evidence by illustration. 
(23) The second example is regarding music genres. Artists are emulating the style of other artists. It is nearly impossible for a new music genre not to have influence from another, at this time when so many already exist. (R2)

(24) For example, Chopin, who is the composer at the romantic era, did not perfectly belong to the romantic form. This can be illustrated by the fact that Mozart's scale considered as a part of classical form is also into Chopin's works. Considering this result, Chopin has developed the romantic music by adopting good points at the classical era and also adding his originality into it. (J1)

Authors from both groups resort, to different extents, to expert opinion, which they introduce by means of evidentials; but only the Japanese writers provide evidence based on personal experience, as well as on factual data derived from statistics, ethos, past events and/or logical connections between them (e.g., comparison and contrast, in J6).

\subsection{Discussion}

The findings introduced in the previous sections show some inter-group variance that deserves further comment. It should be noted at the outset that, by and large, the essays in both corpora largely fit the description of singlefunctionarguments. However, three texts in the Japanese set (J1, J2, J4) and one in the Romanian corpus (R4) show evidence of what might be considered - with some leeway - a dialectical response. Specifically, in these papers, the authors acknowledge the opposite point of view, but do not attempt to challenge it beyond a terse expression of disagreement or, like in the following example, a suggestion of disagreement:

(25) Some people insist that traditional factors always kill something new and suppress those vibes which could be creative or beneficial in the future. However, aren't there any ways to think differently? (J4)

As mentioned earlier (cf. 1.3.2), research in L1 composition has shown that, to all intents and purposes, Japanese language writing is readerresponsible. What this means is that, since meaning-making is the readers' onus, the writer has little obligation to facilitate their access to the intended message, or even acknowledge their presence at all. These native language features did not carry over into the Japanese students' L2 writing, where the high density of metadiscoursal markers and (in some texts) of interactional metalanguage is consistent with writer-responsible prose.

The situation is different with the texts in the Romanian corpus, however. Here the writers' intentions and the logical relations between different assertions are not always immediately apparent to the reader. The 
fact that these texts are, by comparison, more reader-responsible can be put down - at least in part - to rhetorical interference with Romanian language composition which, based on this author's experience, can be describedas predominantly reader-responsible.

In contrast with their native writing practices (cf. 1.3), the Japanese students writing in English are more assertive in communicating their position on the matter in hand; most typically, the thesis is introduced at the beginning of the essay and, from there, the writer proceeds by defending it. On the other hand, the location of the thesis is variable in the papers forming the Romanian corpus, where initial thesis position occurs in just one solitary case. In these texts, the writer's side of the argument is most often implicit, being sometimes embedded in elaborate or verbose expository chunks of text which obscure, rather than clarify, the intended message.

Interestingly enough, in the Japanese group, the high incidence of selfmentions adds to the assertiveness of the claims, signalling the arguer's persona and their commitment to the propositional content of the argument. In the Romanian texts, the comparatively lower profile that the writers keep and their predilection for impersonal, collective, and/or generic pronouns sometimes stands in an almost paradoxical contrast to the subjective bias of theirassertions.

This brings the discussion to another focal point of this investigation: the types of evidence that writers from each group make use of in order to defend their position. It will be remembered that, rather than merit, the objects of interest here have been the amount and variety of evidentiary support. Overall, the number of instances of evidence is higher in the Japanese corpus. Also, with one exception, the Japanese writers deploy more than one type of evidence, a strategy which works well, especially when it is aimed at reaching as wide an audience as possible. The range of evidence types is drastically limited in the Romanian corpus, where illustration is the preferred type. Illustration figures high on the frequency scale in the Japanese corpus, too. But, unlike the Romanian writers, who tend to leave it to the reader to determine its relevance, the students in the Japanese group elaborate on their illustrations more. In these texts, illustrations and explanations may also be embedded in instances of evidence by opinion, which brings them closer to being "considered opinions" (cf. Wingate 2012), as opposed to the often non-verifiable opinions present in the essays from the Romanian corpus.

\section{CONCLUSIONS}

The analysis reported above has provided a brief description of the relative performance of two groups of novice writers responding to the same writing 
task: nine Japanese business students and nine Romanian EL students composing argumentative texts in English, in the context of an out-of-class essay competition. Since the students in the Japanese group were more effective in achieving their communicative purposes, their output has been taken as a reference point in the contrastive investigation of three specific aspects of argument composition i.e., the manner in which the writers signal their intentions via metatext and discourse markers; the way they introduce their Theses; and the types of evidence they advance in order to uphold their claims. This contrastive approach was taken with a view to diagnosing the causes of the subprime performance by the Romanian students. From a wider perspective, the qualitative interpretation of the findings points at two main reasons for the disparities found across the texts in the two subcorpora: one relates to the students' attitude towards the writing task itself and the other, to the different focus of attention with each group. Specifically, the Japanese students approach the written assignment as a real-life argument taking place in a real-life situation; they therefore concentrate on building their case by combining different types of evidentiary support. The Romanian students, on the other hand, treated the writing task as an exercise in composition, attending more to the language and style of their text, rather than to the purpose it was intended to perform. As a result, whilst more elaborate in terms of vocabulary and syntax, their texts were less effective, rhetorically, because of their lower level of readability and the comparatively weaker argumentation patterns. To some extent, the Romanian students' focus on language may echo the preeminent position that language teaching and learning holds in their educational context. Since the construction of argument involves both language skills and cognitive skills, overemphasis on the former does not compensate - at least as thesedatashow - for the deficit in the latter. It may be argued, then, that formal induction in the process of argumentation is likely to help students not only to write better, but also to reason more clearly. Evidence of this are the Japanese students in this study, whose previous instruction in debate has very likely been instrumental in their writing performance.

\section{Works Cited}

Ädel, A. Metadiscourse in L1 and L2 English. Amsterdam/Philadelphia: John Benjamins Publishing Company, 2006. Print.

Browne, N. M., and Keeley, S. M. Asking the Right Questions. A Guide to Critical Thinking. (8th ed.). USA: Pearson Prentice Hall, 2010. Print.

Coffin, C., Curry M. J., Goodman, S., Hewings, A., Lillis T., and Swann, J. Teaching academic writing. New York: Routledge, 2003. Print.

Connor, U. Contrastive Rhetoric. Cross-Cultural Aspects of Second-Language Writing. Cambridge University Press, 1996. Print. 
Glassner, A. "Evaluating arguments in instruction: Theoretical and practical directions”. In Thinking Skills and Creativity 24. Elsevier, 2017. 95-103. Print.

Govier, T. A Practical Study of Argument (7th edition). USA: Wadsworth Cengage Learning, 2010. Print.

Grabe, W. and Kaplan, R.B. Theory and practice of writing. New York: Longman, 1996. Print.

Groarke, L, “Informal Logic”. In The Stanford Encyclopedia of Philosophy (Spring 2017 Edition), Edward N. Zalta (ed.),

URL = <https://plato.stanford.edu/archives/spr2017/entries/logic-informal/>.

Groarke, L. "Going Multimodal: What is a Mode of Arguing and Why Does it Matter?”. In Argumentation. Springer, 2015. 29:133-155. Print.

Hitchcock D. "Informal Logic and the Concept of Argument”. In: On Reasoning and Argument. Argumentation Library, vol 30. Springer, Cham 2017. 447-475. Print.

Hurley, P. J. A Concise Introduction to Logic. (7th edition) Wadsworth Publishing, 2000. Print.

Hyland, Ken “Metadiscourse: Mapping Interactions in Academic Writing”. In Nordic Journal of English Studies, 9(2), 2010. 125-143. Print.

Johnson, R. H. "More on Arguers and Their Dialectical Obligations". OSSA Conference Archive. Paper 65. 2001. http://scholar.uwindsor.ca/ossaarchive/OSSA4/papersandcommentaries/65.

Johnson, R. H. and Blair, J. A. Logical Self-Defense. New York: International Debate Education Association, 2006. Print.

Kuhn, D. and Udell, W. “Coordinating own and other perspectives in argument”. In Thinking \& Reasoning, Taylor and Francis Group. 13 (2), 2007. 90 - 104. Print.

Stapleton, P. and $\mathrm{Wu}, \mathrm{Y}$. “Assessing the quality of arguments in students' persuasive writing: A case study analyzing the relationship between surface structure and substance”. In Journal of English for Academic Purposes 17, 2015. 12-23. Print.

Strongman, L. Academic Writing. Newcastle: Cambridge Scholars Publishing, 2013. Print.

Tadros, A. "Predictive categories in expository writiting”. In M. Coulthard (ed.) Advances in written text analysis. London/N.Y.: Routledge, 1994. 69-82. Print.

Toulmin, S. E. The uses of argument (updated edition). Cambridge: Cambridge University Press, 2003. Print.

Wingate, U. “'Argument!' helping students understand what essay writing is about”. In Journal of English for Academic Purposes 11, 2012. 145-154. Print.

\section{Acknowledgements}

The author gratefully acknowledges the contribution of Prof. Daniela Caluianu, from the EL Department of Otaru University of Commerce in Japan, who initiated and organized the international essay contest leading to the collection of useful contrastive data. 